\title{
Proteomics reveal biomethane production process induced by carbon nanotube
}

\author{
Jian Liu ${ }^{\text {a,1 }}$, Fanghua Liu ${ }^{\text {b,c,1 }}$, Jiafeng Yu ${ }^{\text {a, }}$, Quan Wang ${ }^{\text {d }}$, Zhenkai Li ${ }^{\text {d }}$, Kui Liu ${ }^{\text {a }}$, Congmin Xu ${ }^{\text {a }}$ \\ Hang $\mathrm{Yu}^{\mathrm{b}}$, Leilei Xiao ${ }^{\mathrm{b}, \mathrm{c}, * *}$ \\ a Shandong Key Laboratory of Biophysics, Institute of Biophysics, Dezhou University, Dezhou, 253023, PR China \\ ${ }^{\mathrm{b}}$ Key Laboratory of Coastal Biology and Biological Resources Utilization, Yantai Institute of Coastal Zone Research, Chinese Academy of Sciences, Yantai, 264003, PR \\ China \\ ${ }^{\mathrm{c}}$ CAS Key Laboratory of Coastal Environmental Processes and Ecological Remediation, Yantai Institute of Coastal Zone Research, Chinese Academy of Sciences, Yantai, \\ 264003, PR China \\ ${ }^{\mathrm{d}}$ School of Resources and Environmental Engineering, Ludong University, Yantai, 264025, PR China
}

\section{A R T I C L E I N F O}

\section{Keywords:}

Methanogenic archaea

Methane

Protein analysis

Conductive materials

Pure culture

\begin{abstract}
A B S T R A C T
Biomethane produced by methanogenic archaea is a main greenhouse resource of terrestrial and marine ecosystems, which strongly affects the global environment change. Conductive materials, especially nano-scale, show considerable intervention on biomethane production potential, but the mechanism is still unclear. Herein, we precisely quantified the absolute abundance of Methanosarcina spp. proteins affected by carbon nanotubes (CNTs) using tandem mass tag (TMT) proteomics technology. Among the 927 detectable proteins, more than three hundred, 304, showed differential expression. Gene Set Enrichment Analysis on KEGG pathways and GO biological processes revealed a trend of decreased protein synthesis induced by CNTs, suggesting these conductive nanomaterials may replace part of the cell structure and function. Interestingly, increased acetoclastic methanogenesis actually came at the expense of reduced protein synthesis in related pathways. CNTs stimulated biomethane production from acetate by stimulating intracellular redox activity and the $-\mathrm{COOH}$ oxidation process. These findings enhanced the understanding of the biomethane production process affected by conductive materials.
\end{abstract}

\section{Introduction}

Methane is an active player in the global carbon cycle and serves as renewable fuel with crucial application potential. Biomethane production is an anaerobic process, which is the primary energy obtaining step for methanogenic archaea (Evans et al., 2019). Methanogens, which belong exclusively to the domain Archaea, are globally spread in various environments, such as sediments, anaerobic soils, landfills, wastewater and so on (Xiao et al., 2017, 2021a; Li et al., 2018a; Chen et al., 2020a). Methanogenic archaea have been identified as the main component in many anaerobic digestion systems, which play a crucial role in global carbon cycle and biomethane production (Zhu et al., 2020; Cai et al., 2021). There are three known methanogenesis pathways, and the main biomethane producing pathways are acetoclastic methanogenesis and $\mathrm{CO}_{2}$ reduction, contributing to approximately $2 / 3$ and $1 / 3$ of the global biomethane production, respectively (Conrad, 2005).

For all methanogenic progresses, Mcr and Mtr catalyzing the last two steps of methanogenesis are the crucial components of methanogens. Methanogenic archaea generally contain only one or two methanogenesis pathways, while Methanosarcina spp., cytochrome containing methanogens, produce methane through all three known methanogenic pathways (Mand and Metcalf, 2019). Moreover, Methanosarcina spp. often dominate the biomethane production in many anaerobic digestion systems (Tsapekos et al., 2017; Xiao et al., 2018, 2019a, Kurade et al., 2019). It was reported that Methanosarcina switched electron transport chains according to environmental condition (Mand et al., 2018).

\footnotetext{
* Corresponding author.

** Corresponding author. Key Laboratory of Coastal Biology and Biological Resources Utilization, Yantai Institute of Coastal Zone Research, Chinese Academy of Sciences, Yantai, 264003, PR China.

E-mail addresses: jfyu1979@126.com (J. Yu),11xiao@yic.ac.cn (L. Xiao).

${ }^{1}$ Contribute equally.
} 
Therefore, methanogens evolve many numerous transport proteins such as Ech, Fpo, Vho, Hdr, and so on.

In the past decade, direct interspecies electron transfer (DIET) coupled methanogenesis is considered an important biomethane producing process in complex environments (Morita et al., 2011; Song et al., 2019; Xiao et al., 2019b, c). This is largely due to the participation of some bacteria that can produce electrons, such as Geobacter (Morita et al., 2011; Liu et al., 2012; Xiao et al., 2021b). Conductive materials such as nano-magnetite and carbon nanomaterials in the system can significantly enhance this coupling process (Liu et al., 2012; Xiao et al., 2020a; Chen et al., 2020b). The strengthened electrons transfer from exoelectrogenic bacteria to $\mathrm{CO}_{2}$ reducing archaea was first proposed to explain the enhanced methane production. Nevertheless, it is not clear whether the bacteria or the archaea primarily respond. Recent studies showed that conductive magnetite promoted methane production in pure archaea culture through enhanced acetate dismutation (Fu et al., 2019). Further, we proposed a potential mechanism by which CNTs promoted acetoclastic methanogenesis by increasing intracellular electron transfer (Xiao et al., 2020b). However, the detailed analysis is absent and how methanogen regulates whole protein expression to respond to conductive nanomaterial is still unknown.

In this study, combining proteomic analysis and several statistical tools, we deeply screened the proteins expressed in acetoclastic methanogen under anaerobic condition with or without CNTs. The differentially expressed genes (DEGs) were discovered and the key pathways affected by CNTs were identified. A statistic tool was applied to identify the most stably DEGs which might play a role in biomethane production. Finally, we drawn a general illustration about the biomethane production pathway stimulated by CNTs.

\section{Materials and methods}

\subsection{Methanogens and culture conditions}

The cultivation of pure $M$. barkeri can refer to Xiao et al. (2020b). In brief, $M$. barkeri was inoculated in Mineral salt medium supplied with or without CNTs ( $\sim 0.2 \mathrm{~g} / \mathrm{L}$, Macklin, Shanghai, China; CAS: 308,068-56-6, Lot\#:C10112635, Inner diameter: 5-10 nm, Outer diameter: 10-20 nm, Length: 500-2000 nm) under strict anaerobic conditions in the dark. Acetate was used as the methanogenic substrate with the final concentration of about $20 \mathrm{mmol} \mathrm{L}^{-1}$. The cultivation was performed in anaerobic tubes ( $25 \mathrm{~mL}$ total volume, medium volume of $10 \mathrm{~mL}$ ) pressurized with a mixture of $\mathrm{N}_{2} / \mathrm{CO}_{2}(80 \% / 20 \%)$ with triplicates for each treatment.

\subsection{LC-MS/MS based methanogen proteomics}

Proteome was used to analyze the protein expression of $M$. barkeri. Briefly, the total protein samples were collected on the 25th day. Frozen samples were lysed with lysis buffer (150 mM KCl, $50 \mathrm{mM} \mathrm{HEPES,} 0.1 \%$ CHAPS, $\mathrm{pH}=7.4$ ) supplemented with $1 \mathrm{mM}$ PMSF and sonication (10 times for $10 \mathrm{~s}$ each with $50 \%$ amplitude and $30 \mathrm{~s}$ intervals on ice). Raw protein extract was loaded on a $10 \mathrm{~K}$ ultrafiltration tube with reducing buffer (10 mM DTT, $8 \mathrm{M}$ Urea, $100 \mathrm{mM}$ TEAB, $\mathrm{pH} 8.0$ ) at $60{ }^{\circ} \mathrm{C}$ for $1 \mathrm{~h}$. IAA was then added to the solution with a final concentration of $50 \mathrm{mM}$ for $40 \mathrm{~min}$ at room temperature. The proteins were washed three times with $100 \mathrm{mM}$ triethylammonium bicarbonate (TEAB) and subsequently digested by sequencing-grade trypsin $(1 \mu \mathrm{g} / \mu \mathrm{L})$ in $100 \mathrm{mM}$ TEAB at $37{ }^{\circ} \mathrm{C}$ for $12 \mathrm{~h}$. The digested peptides were collected by centrifugation and lyophilized. The resulted peptides were labeled using a 6-plex TMT reagent Multiplex kit (Applied Biosystems, Foster City, CA) according to the manufacturer's protocol. The labeled peptides from different samples were equally combined and lyophilized.

The combined peptides were dissolved in Milli-Q water containing $0.1 \%$ formic acid (FA) and first subjected to reversed-phase (RP) separation on an Agilent 1100 HPLC System (Agilent Technologies, Palo
Alto, CA, USA) using an Agilent Zorbax Extend RP column (5 $\mu \mathrm{m}, 150$ $\mathrm{mm} \times 2.1 \mathrm{~mm}$ ). Mobile phases A ( $2 \%$ acetonitrile in HPLC water) and B ( $98 \%$ acetonitrile in HPLC water) were used for RP gradient. The solvent gradient was set as follows: 0 8 $\mathrm{min}, 98 \% \mathrm{~A} ; 8.00-8.01 \mathrm{~min}, 98 \%-95 \%$ A; 8.01-38 min, 95\%-75\% A; 38-50 $\mathrm{min}, 75-60 \%$ A; 50-50.01 min, 60-10\% A; 50.01-60 min, 10\% A; 60-60.01 min, 10-98\% A; 60.01-65 $\min , 98 \% \mathrm{~A}$. The fluent flow rate was $300 \mu \mathrm{L} / \mathrm{min}$. The samples were harvested from $8 \mathrm{~min}$ to $50 \mathrm{~min}$, and elution buffers were collected every minute. The separated peptides were lyophilized for LC-MS detection.

The aliquots were dissolved in $0.1 \%$ formic acid solution and analyzed by an LC-MS system consisting of an Ultimate3000 system (Thermo, USA) and a Q-Exactive HF mass spectrometer (Thermo, USA) equipped with a Nanospray Flex source (Thermo, USA). Samples were loaded and separated by a C18 column $(15 \mathrm{~cm} \times 75 \mu \mathrm{m})$. The flow rate was $250 \mathrm{~nL} / \mathrm{min}$, and linear gradient was set as follows: $5-8 \%$ B over $7 \mathrm{~min}, 8-30 \%$ B over $55 \mathrm{~min}, 30-50 \%$ B over $24 \mathrm{~min}, 50-90 \%$ B over 2 min (mobile phase A containing $0.1 \% \mathrm{FA}$ in water and phase B containing $0.1 \%$ FA in acetonitrile). Full MS scans were acquired in the mass range of $300-1500 \mathrm{~m} / \mathrm{z}$ with a mass resolution of 120,000 , and the AGC target value was set at 3e6. The 12 most intense peaks in MS1 were fragmented with higher-energy collisional dissociation (HCD) with a collision energy of 32. MS/MS spectra were obtained with a resolution of 45,000 , an AGC target of $1 \mathrm{e} 5$, and a max injection time of $100 \mathrm{~ms}$. The QExactive HF dynamic exclusion was set for $30.0 \mathrm{~s}$ and run under positive mode.

ProteomeDiscoverer (v.2.2) was used to search all the raw data against the proteome of M. barkeri (strain Fusaro/DSM 804) obtained from the UniprotKB database. A global false discovery rate (FDR) was set to 0.01 , and protein groups considered for quantification required at least 2 peptides.

\subsection{Differentially expressed gene analysis}

The package limma (version 3.46.0) implemented in $\mathrm{R}$ (version 4.0.3) was applied to identify differentially expressed genes (DEGs). As described previously, one sample of the CNTs group was removed from the analysis because of obvious experimental contamination. With the "ImFit" function, we built a general linear model analysis on all the quantified proteins. With the "eBayes" function, we performed an empirical Bayes inference to analyze the differences between the two groups. The criteria of a fold change larger than 1.5 and adjusted p-value less than 0.05 was set to select proteins with a significant expression difference.

\subsection{Go and KEGG analysis}

The GO and KEGG analyses were conducted using the R package clusterProfiler (Yu et al., 2012, version 3.18.0). The KEGG annotations were obtained through its application programming interface, and the GO annotations were obtained from the UniprotKB database. We manually constructed the background package of $M$. barkeri using the $\mathrm{R}$ package AnnotationForge (version 1.32.0). Up-regulated genes and down-regulated genes were applied for over-representation analysis separately, and the overall ordered expression profile was applied for gene set enrichment analysis with default parameters.

\subsection{Expression variation analysis}

To assess the stability of the gene expression, we calculated the differences between samples from each group. The mean difference and the standard deviation of the six comparisons were then obtained and plotted. 
A

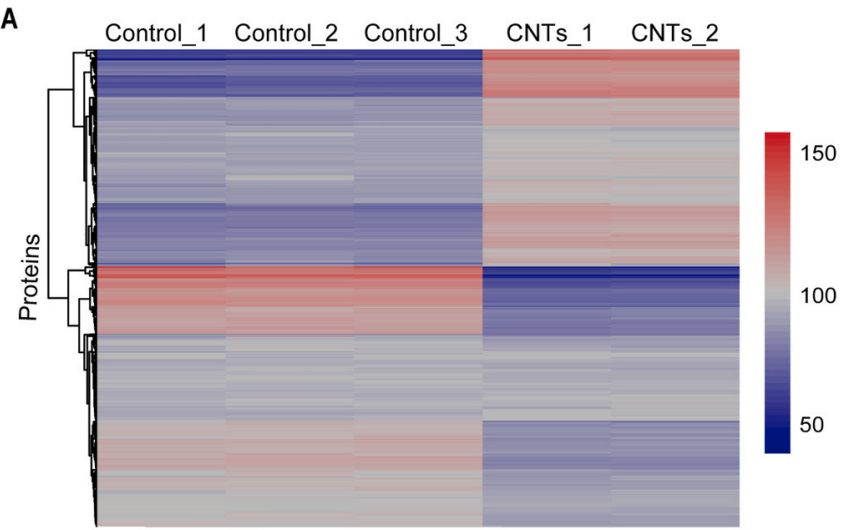

B

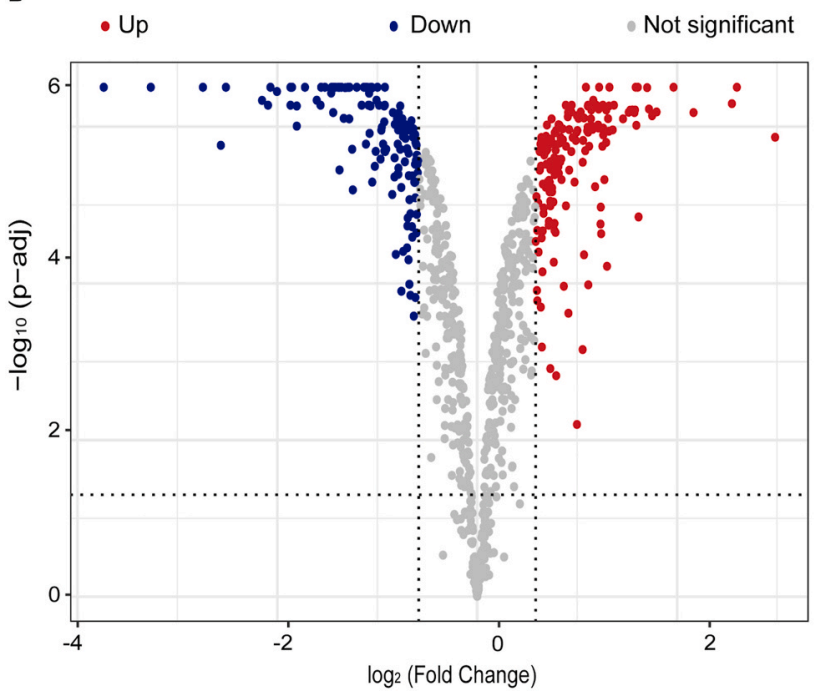

Fig. 1. The overall expression pattern. A: heat map of the expression level for all quantified proteins. Each line presented a protein; each column presented a sample. The proteins were clustered by the hclust function. B: volcano plot for DEG analysis with $\mathrm{x}$-axis showed the log base 2 of the fold changes and $y$-axis showed the log base 10 of the adjusted p-value. Grey points represented not significant proteins, blue and red points represented down-regulated and upregulated genes, respectively. The dash lines represent the selection criteria of fold change and adjusted p-value. (For interpretation of the references to color in this figure legend, the reader is referred to the Web version of this article.)

Table 1

Summary of the proteomic analysis.

\begin{tabular}{llll}
\hline & All & Function identified & Function Unknown \\
\hline Identified protein & 927 & 715 & 212 \\
Quantified protein & 849 & 714 & 135 \\
Differential protein & 304 & 225 & 79 \\
Increased protein & 164 & 107 & 57 \\
Decreased protein & 140 & 115 & 25 \\
\hline
\end{tabular}

\section{Results and discussion}

\subsection{CNTs largely changed the global expression pattern}

Using labeling LC-MS/MS proteomics analysis method, we identified 927 individual proteins; 849 of them were successfully quantified in all the five samples (Tables 1 and S1), which present $23.5 \%$ of the 3616 proteins reported from the reference genome (Maeder et al., 2006). Obviously, the two groups presented different expression patterns (Fig. 1A). Compared to the control treatment, the proportion of
A

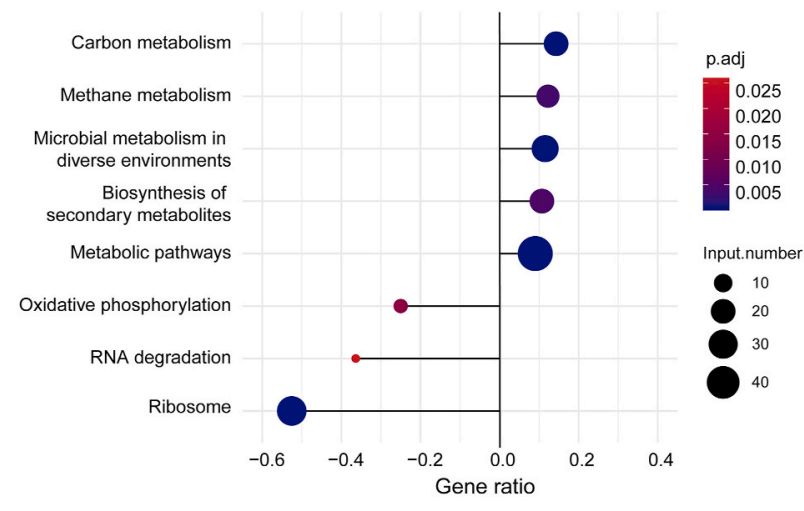

B

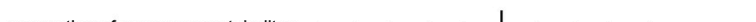

generation of precursor metabolites anaerobic respiration methane metabolic process methanogenesis methanogenesis
energy derivation by oxidation of reduced inorganic compounds
cellular alkane metabolic process alkane biosynthetic process cellular respiration cellular respiration
energy derivation by oxidation of organic compounds
idation-reduction process biological_process biosynthetic process cellular process metabolic process organic substance metabolic process cellular biosynthetic process organic substance biosynthetic process cellular metabolic process organonitrogen compound metabolic process nitrogen compound metabolic process ion transport organonitrogen compound biosynthetic process
cation transport response to stress primary metabolic process monovalent inorganic cation transport cellular nitrogen compound metabolic process cellular nitrogen compound biosynthetic process
gene expression

macromolecule metabolic process macromolecule biosynthetic process protein metabolic process cellular macromolecule biosynthetic process amide biosynthetic process

cellular amide metabolic process cellular protein metabolic process cellular macromolecule metabolic process peptide metabolic process peptide biosynthetic process

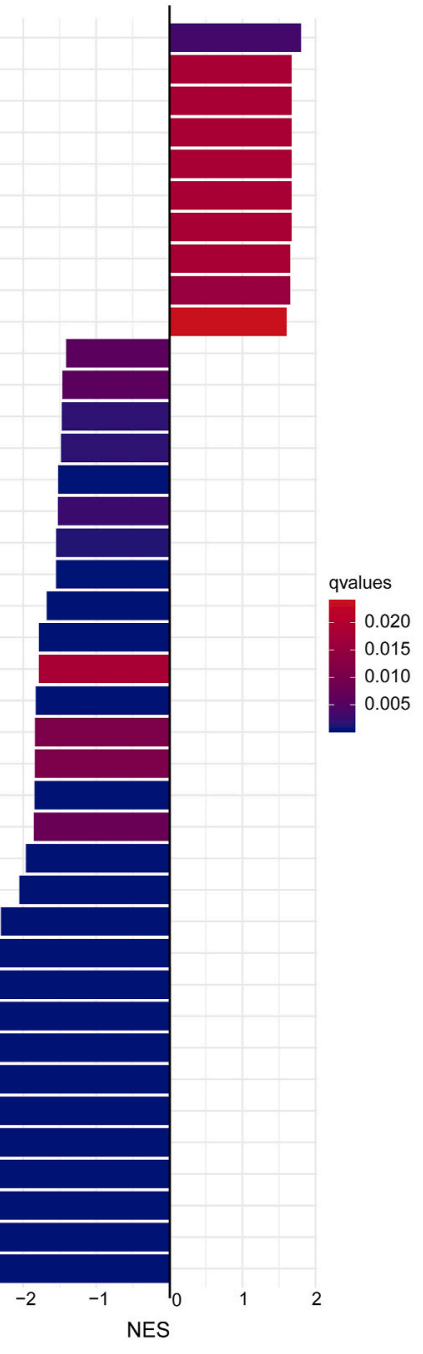

Fig. 2. KEGG and GO analysis. A: KEGG pathway over-representation analysis. The absolute value of the y axis represents the gene ratio of DEGs in the specific pathway. Positive value and negative value represent up-regulated and downregulated, respectively. The circle size represents the number of DEGs; the color of the circle represents the adjusted p-value. B: GO biological process gene set enrichment analysis. The $\mathrm{x}$-axis represents the enrichment score, positive value and negative value represent up-regulated and down-regulated, respectively. The color of the bar represents the adjusted p-value. (For interpretation of the references to color in this figure legend, the reader is referred to the Web version of this article.) 
A

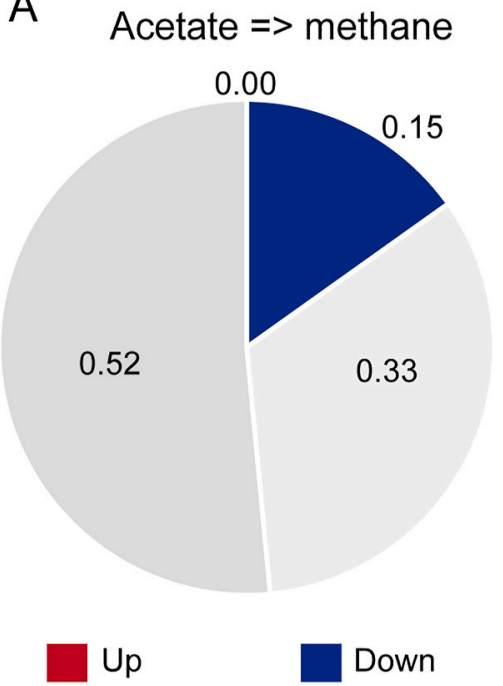

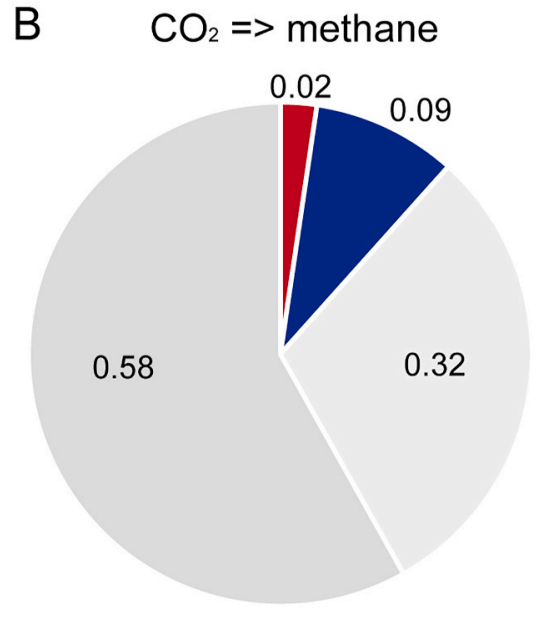

Not significant
Not quantified

Fig. 3. The proportion of different genes in two KEGG methanogenesis module. A: acetate to methane module. $\mathrm{B}$ : $\mathrm{CO}_{2}$ to methane module.

decreased proteins was larger than increased ones, indicating a global protein synthesis decreased.

We identified 304 DEGs, 79 of which are unknown functions at present (Table 1, S2), indicating the importance of unknown proteins to biomethane production (He et al., 2019). The proportion of unknown proteins in DEGs is similar to that of the genome (1200/3616, Maeder et al., 2006), showing the need for more detailed functional annotation on the genome of $M$. barkeri. Among the DEGs, 140 proteins amount significantly decreased while 164 proteins significantly increased (Table S2, Fig. 1B). Although CNTs promoted more protein synthesis, the decreased proteins showed a larger fold change. Especially, $50 \mathrm{~S}$ ribosomal proteins (L44e, L37e) showed extremely decreased expression with a fold change smaller than 1/10. Recently, Yan et al. (2020) found that zero-valent iron and magnetite nanoparticles could decrease the ribosome protein levels in anaerobic digestion sludge, but the decreasing attitude is lower than the presented results. In contrast, Yan et al. (2020) found that powder activated carbon could increase ribosome protein levels. Thus, the effects of ribosomes to biomethane production may depend on the characteristics of the conductive materials.

In comparison, dimethylamine corrinoid proteins are the most increased proteins with known functions. They are essential components of the methanogenic pathway, which could produce $\mathrm{Na}^{+}$gradient for energy conservation but not directly participate in the acetoclastic methanogenesis but methylotrophic methanogenesis (Kurth et al., 2020). Previously detailed evidence confirmed that CNTs promoted acetoclastic methanogenesis (Xiao et al., 2020b). A larger $\mathrm{Na}^{+}$gradient and more energy production may benefit acetate dismutation for biomethane generation.

\subsection{CNTs served as structural proteins}

Fig. 2A showed the over-representation analysis of the KEGG pathways. The methanogenesis pathway was over-represented in upregulated genes, which was also observed in the previous study ( $\mathrm{He}$ et al., 2019) with enhanced methane production. However, the ribosome pathway was over-represented in down-regulated genes. Thus, the overall protein synthesis was decreased in the CNTs group. It was suggested that under stressful conditions, cells often decreased protein synthesis to minimize energy consumption (Yan et al., 2020). Fujinawa et al. (2019) showed that high concentration carbon nanoparticles inhibited the growth of archaea because of their antimicrobial activity and decreased biomethane production. On the contrary, a previous study showed that the CNTs attached to the cell surface and promoted methane production (Xiao et al., 2020b). Meanwhile, CNTs often showed good potential to combine with proteins and increased the activity of the attached proteins (Li et al., 2018b). Therefore, the CNTs attached to the cell wall to serve as structural proteins and promote the activity of surface proteins, which may decrease the overall protein expression.

Fig. 2B showed the gene-set enrichment analysis of the GO biological process. The methanogenesis was enriched for increased expression, while the translation, gene expression, and peptide synthesis processes were enriched for decreased expression. These results were constant with the KEGG analysis. Intriguingly, ion transport and nitrogen compound metabolic process showed a significant decrease. In contrast, a previous study showed that an increase of acetate concentration enhanced methane production through enhanced ion transport and nitrogen metabolism, which related to electron transfer and protein activity (He et al., 2019). In complex anaerobic digestion system, CNTs were shown to enhance methane production through improved DIET (Mostafa et al., 2020). But the present results implied an enhanced electron transfer efficiency within the cell by CNTs. As we mentioned before, CNTs could act as structural proteins; thus, it could increase methane production without increased ion transport and nitrogen metabolism.

Further analysis of the KEGG modules showed that both the $\mathrm{CO}_{2}$ to methane module and the acetate to methane module had more downregulated genes than that of up-regulation (Fig. 3). The methanogenesis process in complex environments could be enhanced by many different materials, such as magnetite, iron, activated carbon, hydrochar, graphite, and so on. These materials can increase the proportion of acetoclastic methanogens such as Methanosarcina spp. and Methanothrix spp. (Tang et al., 2020; Du et al., 2020). A study showed that CNTs promoted acetoclastic methanogenesis (Xiao et al., 2020b). Therefore, the increase of specific essential proteins may be the critical point of the promoting effect of CNTs on the acetate utilization. In contrast, it is not required to increase the whole pathway's protein level or even most of the pathway. This strategy also helps to conserve material and energy consumption.

\subsection{CNTs altered electron transfer system}

Crucial genes generally exhibit stable expression (Sun et al., 2013), therefore we calculated the differences between every two samples from each group (Table S3) to analyze the expression variation of the proteins. The mean difference and the standard deviation of the differences 


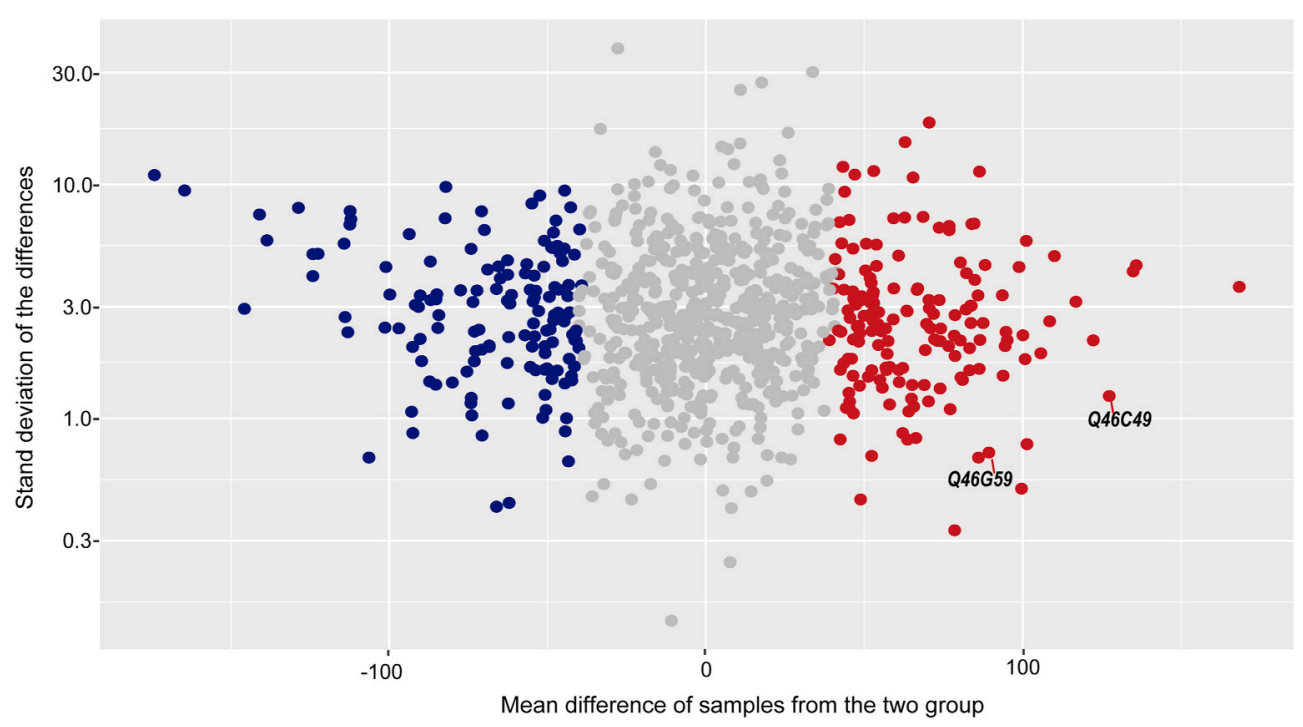

Fig. 4. The mean difference (x-axis) and standard deviation (y-axis) of proteins between every two samples from each group. Grey points represent not significantly changed genes. Blue points and red points represent up-regulated and down-regulated genes, respectively. (For interpretation of the references to color in this figure legend, the reader is referred to the Web version of this article.)

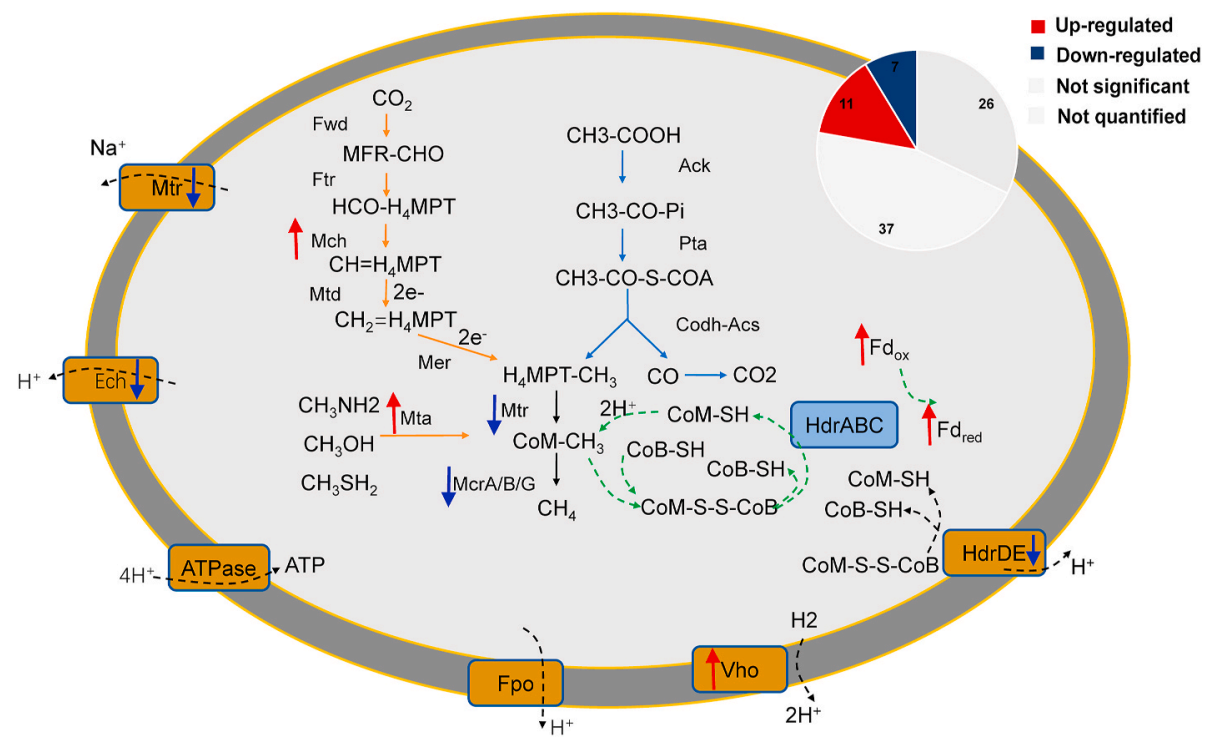

Fig. 5. Summary of the methanogenesis pathways affected by carbon nanotubes. Significant up-regulation and down-regulation are shown with red and blue arrows, respectively. The pie chart on the top-right showed the number of all detectable proteins. (For interpretation of the references to color in this figure legend, the reader is referred to the Web version of this article.)

are plotted in Fig. 4. The points far from the central point indicate large differences, and the points on the bottom express fewer variations among the replicates. For the down-regulated proteins, 31 of them are ribosome proteins, showing a strong depression of protein synthesis. Fujinawa et al. (2019) showed that a high concentration of conductive materials could decrease protein synthesis and methane production due to their toxicity.

Except for the genes discussed before, two genes, Q46C49 (Sulfite reductase, assimilatory-type) and Q46A58 (Thioredoxin), showed large increases in the CNTs group with considerable low variations. It suggested their potential function for acetoclastic methanogenesis. Previous studies demonstrated both two kinds of proteins are redox proteins that participated in the resistance to oxidative stress (Yu et al., 2018). However, no study confirmed their effective function on methanogenic progress. We suppose that these proteins contributed to the electron transfer process for effective methane production.
The overall methanogenesis pathways were summarized in Fig. 5. Only a few proteins are significantly regulated, showing their importance during the production of methane. Among the electron transfer proteins, only one of the Vho/Vht proteins was up-regulated. Analysis using deficient mutants showed that Vho is indispensable for $M$. barkeri to grow on acetate (Kulkarni et al., 2018; Mand et al., 2018). Concerning that Ech was decreased by CNTs, CNTs may attach to the surface protein Vho and increase their activity, consequently, enhance the overall electron transfer. Moreover, ferredoxin involved in the recovery of $\mathrm{CoM} / \mathrm{CoB}$, which participate the function of the crucial enzyme Mcr, were increased. Within complex anaerobic digestion system, proteomic analysis identified similar increase of ferredoxin in a biomethane increasing condition (Lam et al., 2021). Collectively, CNTs may stimulate biomethane production from acetate by intracellular redox activity and the $-\mathrm{COOH}$ oxidation process. 


\section{Conclusion}

The finding of this research revealed the molecular mechanism of the increased acetoclastic methane production induced by CNTs, which is proved by previous detailed evidence. Serving as structure protein, increasing intracellular redox activity and the $-\mathrm{COOH}$ oxidation process mainly attributed to high methane production performance. These results enrich the understanding of the biomethane production process in anaerobic environments affected by conductive nanomaterials.

\section{Sources of funding}

This work was supported by the National Natural Science Foundation of China (61771093, 42077025, U20A20109, 62011530044), Youth Innovation Promotion Association, CAS (2021213), the Youth Science and Technology Innovation Plan of Universities in Shandong (2019KJE007), and Natural Science Foundation of Shandong Province (ZR2016JL027).

\section{Credit author statement}

Jian Liu: Methodology, Visualization, Formal analysis, Data Curation, Writing - Original Draft, Fanghua Liu: Validation, Resources, Formal analysis, Funding acquisition, Writing - Review \& Editing, Jiafeng Yu: Conceptualization, Methodology, Writing - Review \& Editing, Supervision, Funding acquisition, Quan Wang: Investigation, Data Curation. Zhenkai Li: Investigation, Data Curation. Kui Liu: Investigation, Data Curation. Congmin Xu: Investigation, Data Curation. Hang Yu: Investigation, Data Curation. Leilei Xiao: Conceptualization, Methodology, Writing - Review \& Editing, Project administration, Funding acquisition.

\section{Declaration of competing interest}

The authors declare that they have no known competing financial interests or personal relationships that could have appeared to influence the work reported in this paper.

\section{Appendix A. Supplementary data}

Supplementary data to this article can be found online at https://doi. org/10.1016/j.envres.2021.111417.

\section{References}

Cai, Y., Zheng, Z., Wang, X., 2021. Obstacles faced by methanogenic archaea originating from substrate-driven toxicants in anaerobic digestion. J. Hazard Mater. 403, 123938. https://doi.org/10.1016/j.jhazmat.2020.123938.

Chen, H., Zhu, T., Li, B., Fang, C., Nie, M., 2020a. The thermal response of soil microbial methanogenesis decreases in magnitude with changing temperature. Nat. Commun. 11, 5733. https://doi.org/10.1038/s41467-020-19549-4.

Chen, Q., Liu, C., Liu, X., Sun, D., Li, P., Qiu, B., Dang, Y., Karpinski, N.A., Smith, J.A., Holmes, D.E., 2020b. Magnetite enhances anaerobic digestion of high salinity organic wastewater. Environ. Res. 189, 109884. https://doi.org/10.1016/j. envres.2020.109884.

Conrad, R., 2005. Quantification of methanogenic pathways using stable carbon isotopic signatures: a review and a proposal. Org. Geochem. 36, 739-752. https://doi.org/ 10.1016/j.orggeochem.2004.09.006.

Du, J., Gu, M., Yin, Q., Wu, G., 2020. Temporary addition of carbon fibers facilitates methanogenic degradation of ethanol during anaerobic treatment. Sci. Total Environ. https://doi.org/10.1016/j.scitotenv.2020.142724, 2020, 142724.

Evans, P.N., Boyd, J.A., Leu, A.O., Woodcroft, B.J., Parks, D.H., Hugenholtz, P., Tyson, G. W., 2019. An evolving view of methane metabolism in the Archaea. Nat. Rev. Microbiol. 17, 219-232. https://doi.org/10.1038/s41579-018-0136-7.

Fu, L., Zhou, T., Wang, J., You, L., Lu, Y., Yu, L., Zhou, S., 2019. $\mathrm{NanoFe}_{3} \mathrm{O}_{4}$ as solid electron shuttles to accelerate acetotrophic methanogenesis by Methanosarcina barkeri. Front. Microbiol. 10, 388. https://doi.org/10.3389/fmicb.2019.00388

Fujinawa, K., Nagoya, M., Kouzuma, A., Watanabe, K., 2019. Conductive carbon nanoparticles inhibit methanogens and stabilize hydrogen production in microbial electrolysis cells. Appl. Microbiol. Biotechnol. 103, 6385-6392. https://doi.org/ 10.1007/s00253-019-09946-1.
He, P., Duan, H., Han, W., Liu, Y., Shao, L., Lü, F., 2019. Responses of Methanosarcina barkeri to acetate stress. Biotechnol. Biofuels 12, 289. https://doi.org/10.1186/ s13068-019-1630-5.

Kulkarni, G., Mand, T.D., Metcalf, W.W., 2018. Energy conservation via hydrogen cycling in the methanogenic archaeon Methanosarcina barkeri. mBio 9. https://doi. org/10.1128/mBio.01256-18 e01256-18.

Kurade, M.B., Saha, S., Salama, E.S., Patil, S.M., Govindwar, S.P., Jeon, B.H., 2019. Acetoclastic methanogenesis led by Methanosarcina in anaerobic co-digestion of fats, oil and grease for enhanced production of methane. Bioresour. Technol. 272, 351-359. https://doi.org/10.1016/j.biortech.2018.10.047.

Kurth, J.M., Op den Camp, H.J.M., Welte, C.U., 2020. Several ways one goal-methanogenesis from unconventional substrates. Appl. Microbiol. Biotechnol. https://doi.org/10.1007/s00253-020-10724-7.

Lam, T.K., Yang, J.T., Lai, S.J., Liang, S.Y., Wu, S.H., 2021. Meta-proteomics analysis of microbial ecosystem during the anaerobic digestion of chicken manure in biogas production farm. Bioresource Technology Reports 13, 100643. https://doi.org/ 10.1016/j.biteb.2021.100643.

Li, J., Xiao, L., Zheng, S., Zhang, Y., Luo, M., Tong, C., Xu, H., Tan, Y., Liu, J., Wang, O., Liu, F., 2018a. A new insight into the strategy for methane production affected by conductive carbon cloth in wetland soil: beneficial to acetoclastic methanogenesis instead of $\mathrm{CO}_{2}$ reduction. Sci. Total Environ. 643, 1024-1030. https://doi.org/ 10.1016/j.scitotenv.2018.06.271.

Li, K., Wang, J., He, Y., Abdulrazaq, M.A., Yan, Y., 2018b. Carbon nanotube-lipase hybrid nanoflowers with enhanced enzyme activity and enantioselectivity. J. Biotechnol. 281, 87-98. https://doi.org/10.1016/j.jbiotec.2018.06.344.

Liu, F., Rotaru, A.E., Shrestha, P.M., Malvankar, N.S., Nevin, K.P., Lovley, D.R., 2012. Promoting direct interspecies electron transfer with activated carbon. Energy Environ. Sci. 5, 8982-8989. https://doi.org/10.1039/c2ee22459c.

Maeder, D.L., Anderson, I., Brettin, T.S., Bruce, D.C., Gilna, P., Han, C.S., Lapidus, A., Metcalf, W.W., Saunders, E., Tapia, R., Sowers, K.R., 2006. The Methanosarcina barkeri genome: comparative analysis with Methanosarcina acetivorans and Methanosarcina mazei reveals extensive rearrangement within methanosarcinal genomes. J. Bacteriol. 188, 7922-7931. https://doi.org/10.1128/JB.00810-06.

Mand, T.D., Kulkarni, G., Metcalf, W.W., 2018. Genetic, biochemical, and molecular characterization of Methanosarcina barkeri mutants lacking three distinct classes of hydrogenase. J. Bacteriol. 200 https://doi.org/10.1128/JB.00342-18 e00342-18.

Mand, T.D., Metcalf, W.W., 2019. Energy conservation and hydrogenase function in methanogenic archaea, in particular the genus Methanosarcina. Microbiol. Mol. Biol. Rev. 83 https://doi.org/10.1128/mmbr.00020-19 e00020-19.

Morita, M., Malvankar, N.S., Franks, A.E., Summers, Z.M., Giloteaux, L., Rotaru, A.E., Rotaru, C., Lovleya, D.R., 2011. Potential for direct interspecies electron transfer in methanogenic wastewater digester aggregates. mBio 2. https://doi.org/10.1128/ mBio.00159-11 e00159-11.

Mostafa, A., Im, S., Song, Y.-C., Kang, S., Kim, D.-H., 2020. Enhanced anaerobic digestion of long chain fatty acid by adding magnetite and carbon nanotubes. Microorganisms 8, 333. https://doi.org/10.3390/microorganisms8030333.

Song, X., Liu, J., Jiang, Q., Zhang, P., Shao, Y., He, W., Feng, Y., 2019. Enhanced electron transfer and methane production from low-strength wastewater using a new granular activated carbon modified with nano- $\mathrm{Fe}_{3} \mathrm{O}_{4}$. Chem. Eng. J. 374, 1344-1352. https://doi.org/10.1016/j.cej.2019.05.216.

Sun, L.L., Xiao, L.L., Xiao, B., Wang, W.Y., Pan, C., Wang, S.J., Lian, B., 2013. Differences in the gene expressive quantities of carbonic anhydrase and cysteine synthase in the weathering of potassium-bearing minerals by Aspergillus niger. Sci. China Earth Sci. 56, 2135-2140. https://doi.org/10.1007/s11430-013-4704-4.

Tang, Y., Li, Y., Zhang, M., Xiong, P., Liu, L., Bao, Y., Zhao, Z., 2020. Link between characteristics of $\mathrm{Fe}(\mathrm{III})$ oxides and critical role in enhancing anaerobic methanogenic degradation of complex organic compounds. Environ. Res. 2020, 110498. https://doi.org/10.1016/j.envres.2020.110498.

Tsapekos, P., Kougias, P.G., Treu, L., Campanaro, S., Angelidaki, I., 2017. Process performance and comparative metagenomic analysis during co-digestion of manure and lignocellulosic biomass for biogas production. Appl. Energy 185, 126-135. https://doi.org/10.1016/j.apenergy.2016.10.081.

Xiao, L.L., Xie, B.H., Liu, J.C., Zhang, H.X., Han, G.X., Wang, O.M., Liu, F.H., 2017. Stimulation of long-term ammonium nitrogen deposition on methanogenesis by Methanocellaceae in a coastal wetland. Sci. Total Environ. 595, 337-343. https://doi. org/10.1016/j.scitotenv.2017.03.279.

Xiao, L., Liu, F., Liu, J., Li, J., Zhang, Y., Yu, J., Wang, O., 2018. Nano-Fe $\mathrm{O}_{4}$ particles accelerating electromethanogenesis on an hour-long timescale in wetland soil. Environ. Sci.: Nano 5, 436-445. https://doi.org/10.1039/c7en00577f.

Xiao, L., Liu, F., Xu, H., Feng, D., Liu, J., Han, G., 2019a. Biochar promotes methane production at high acetate concentrations in anaerobic soils. Environ. Chem. Lett. 17, 1347-1352. https://doi.org/10.1007/s10311-019-00863-3.

Xiao, L.L., Sun, R., Zhang, P., Zheng, S.L., Tan, Y., Li, J.J., Zhang, Y.C., Liu, F.H., 2019b. Simultaneous intensification of direct acetate cleavage and $\mathrm{CO}_{2}$ reduction to generate methane by bioaugmentation and increased electron transfer. Chem. Eng. J. 378, 122229. https://doi.org/10.1016/j.cej.2019.122229.

Xiao, L.L., Wei, W.C., Luo, M., Xu, H.D., Feng, D.W., Yu, J.F., Huang, J.F., Liu, F.H., 2019c. A potential contribution of a Fe(III)-rich red clay horizon to methane release: biogenetic magnetite-mediated methanogenesis. Catena 181, 104081. https://doi. org/10.1016/j.catena.2019.104081.

Xiao, L., Liu, F., Lichtfouse, E., Zhang, P., Feng, D., Li, F., 2020a. Methane production by acetate dismutation stimulated by Shewanella oneidensis and carbon materials: an alternative to classical $\mathrm{CO}_{2}$ reduction. Chem. Eng. J. 389, 124469. https://doi.org/ 10.1016/j.cej.2020.124469. 
Xiao, L., Zheng, S., Lichtfouse, E., Luo, M., Tan, Y., Liu, F., 2020b. Carbon nanotubes accelerate acetoclastic methanogenesis: from pure cultures to anaerobic soils. Soil Biol. Biochem. 150, 107938. https://doi.org/10.1016/j.soilbio.2020.107938.

Xiao, L., Wang, Y., Lichtfouse, E., Li, Z., Kumar, S., Liu, J., Feng, D., Yang, Q., Liu, F., 2021a. Effect of antibiotics on the microbial efficiency of anaerobic digestion of wastewater: a review. Front. Microbiol. 11, 611613. https://doi.org/10.3389/ fmicb.2020.611613.

Xiao, L., Li, J., Lichtfouse, E., Li, Z., Wang, Q., Liu, F., 2021b. Augmentation of chloramphenicol degradation by Geobacter-based biocatalysis and electric field. J. Hazard Mater. 410, 124977.

Yan, W., Mukherjee, M., Zhou, Y., 2020. Direct interspecies electron transfer (DIET) can be suppressed under ammonia-stressed condition - reevaluate the role of conductive materials. Water Res. 183, 116094. https://doi.org/10.1016/j.watres.2020.116094.
Yu, G., Wang, L.G., Han, Y., He, Q.Y., 2012. ClusterProfiler: an R package for comparing biological themes among gene clusters. OMICS A J. Integr. Biol. 16, 284-287. https://doi.org/10.1089/omi.2011.0118.

Yu, H., Susanti, D., McGlynn, S.E., Skennerton, C.T., Chourey, K., Iyer, R., Scheller, S., Tavormina, P.L., Hettich, R.L., Mukhopadhyay, B., Orphan, V.J., 2018. Comparative genomics and proteomic analysis of assimilatory sulfate reduction pathways in anaerobic methanotrophic archaea. Front. Microbiol. 9, 2917. https://doi.org/ 10.3389/fmicb.2018.02917.

Zhu, X., Campanaro, S., Treu, L., Seshadri, R., Ivanova, N., Kougias, P.G., Kyrpides, N., Angelidaki, I., 2020. Metabolic dependencies govern microbial syntrophies during methanogenesis in an anaerobic digestion ecosystem. Microbiome 8, 22. https://doi. org/10.1186/s40168-019-0780-9. 